\title{
About the Editor
}
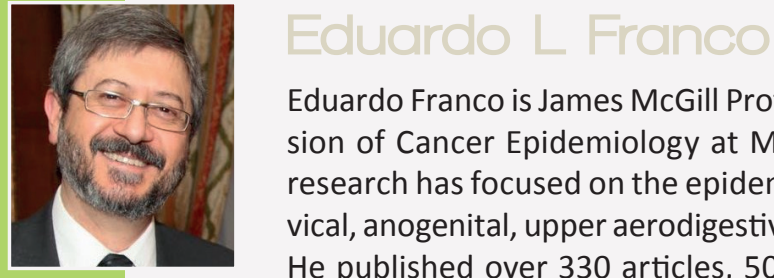

Eduardo Franco is James McGill Professor and Director of the Division of Cancer Epidemiology at McGill University (Canada). His research has focused on the epidemiology and prevention of cervical, anogenital, upper aerodigestive tract and childhood cancers. He published over 330 articles, 50 chapters and two books. He has served in the editorial boards of numerous scientific journals. His awards include: Fellow of the Royal Society of Canada (2011); McLaughlin-Gallie Award, Royal College of Physicians and Surgeons of Canada (2011); Lifetime Achievement Award, American Society for Colposcopy and Cervical Pathology (2010); Honorary President, EUROGIN Congress, Monaco (2010); Women in US Government's Presidential Leadership Award (2008); Canadian Cancer Society's Warwick Prize in CancerControl Research (2004); Medical Research Council of Canada's Distinguished Scientist (2000); Educational Excellence at McGill University (2000); and twice Montreal's 'Ambassadeur' (2000 and 2007). 


\section{Foreword}

\section{Human papillomavirus vaccines}

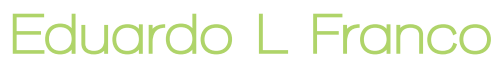

It has been barely 5 years since medicine and public health gained an extraordinary new weapon for preventing cervical cancer, one of the most important neoplastic diseases of women worldwide and a major cause of premature female death in developing countries. The two commercially available vaccines against human papillomavirus (HPV) infection are very effective in preventing infections with the two major cancer-causing genotypes, HPV-16 and HPV-18, and the cervical precancerous lesions that originate from such infections. The clinical trial data are among the strongest of any new vaccines, their safety meets today's standards of low tolerance to risk of adverse events, and protection seems to extend at least to 10 years and probably well beyond. One of the approved vaccines adds protection against two other HPV genotypes (HPV-6 and HPV-11) that cause genital warts and respiratory papillomatosis, diseases that bring substantial suffering and stigmatization to those affected. Both vaccines seem to protect also against other HPV genotypes that are closely related phylogenetically to HPV-16 and -18 , although the duration of that protection remains to be established. Clinical trials of men and mid-adult women have also shown promising results. At least one country, Australia (an early adopter of publicly funded HPV vaccination), has already begun to reap the benefits of HPV vaccination via reductions in rates of cervical abnormalities and genital warts. 
All of the above features of HPV vaccination are well covered in this collection of expertly written chapters. The authors are among the most knowledgeable clinical and basic scientists in the fields of HPV immunology and pathogenesis, epidemiology and infectious diseases. Margaret Stanley begins by providing the critical backdrop on the underpinnings of HPV pathogenesis and its impact on host defensive mechanisms. Suzanne Garland follows with an in-depth account of the results from randomized controlled trials of the quadrivalent vaccine, Merck's Gardasi ${ }^{\circledR}$, the first HPV vaccine to be incorporated into immunization programs. Tino Schwarz does the same for the bivalent HPV vaccine, GlaxoSmithKline's Cervarix ${ }^{\circledR}$, which despite being approved later already enjoys a substantial public health penetration. Readers will appreciate the summaries of the voluminous Phase II and Phase III clinical trial findings and the ancillary data that helps our understanding of the similarities and differences between both vaccines.

Xavier Bosch adds a critical public health perspective on the successes of HPV vaccination. He discusses the complementarity of vaccination to cervical cancer screening, in itself an area that has benefited from the discovery that HPV infection is the central cause of this disease. The social dimensions are central to his argument that the vaccines must become affordable enough to reach those who need it the most, that is, populations in high-fertility, resource-deprived countries in sub-Saharan Africa, Latin America and Southeast Asia. Careful public health planning is also crucial for properly deploying the benefits of HPV vaccination.

Prophylactic HPV vaccination with the main viral capsid L1 works best when administered before sexual exposure to HPV. The two aforementioned vaccines do not appear to protect against existing infections. They will not help the immune system to clear infections by the vaccine-targeted genotypes, nor will they help to eradicate lesions originated from such infections, that is, they do not have therapeutic effects. The pursuit for an HPV vaccine that can attain the latter objective was the focus of the last chapter in this book. Shreya Kanodia and Martin Kast provide the rationale for therapeutic HPV vaccination, an area that has seen much progress but with no concrete candidate vaccines. The authors outlined the lessons that have been learned, all of which require a better understanding of ways to circumventing the immune tolerance induced by established HPV infections.

Although promising, many issues remain concerning prophylactic HPV vaccination. Cervical cancer control programs in many countries have for decades dealt with one tool only, screening with the Pap cytology test. Within these programs, entire professional careers have been devoted to perfecting the delivery of effective cervical cancer screening. While this 
pursuit has been attained in a few resource-rich countries, effective cervical cancer screening with Pap cytology has been an oxymoron for the vast majority. Moreover, vaccination is the jurisdiction of immunization programs, which represent totally different sectors in ministries of health or like agencies. Not surprisingly, there has been resistance against HPV vaccination in some countries, borne of the fear that the locus of control may shift away from delivery of Pap cytology screening. An often-heard argument is that there is no proof that HPV vaccination can reduce incidence of and mortality from cervical cancer. Yet those that voice this concern are the same individuals that propose that Pap cytology screening prevents cervical cancer because it permits the discovery and treatment of precancerous cervical cancer lesions. That HPV vaccination prevents these same lesions and thus would attain the same ultimate dividend of preventing cervical cancer seems to escape these critics.

The issue of high vaccine cost is also frequently raised. As this book went to press, the cost of HPV vaccination had reached US\$15 per dose (from the original price of US\$100-120 when the first vaccine was approved 5 years ago), with potential for further reductions if more countries pooled together with oversight by agencies such as the WHO and Pan American Health Organization. As of June 2011, Merck had announced that it would provide Gardasil at US\$5 per dose for the Global Alliance for Vaccines and Immunization, a consortium that sponsors immunization for the poorest countries of the world. Therefore, vaccine cost is no longer a key obstacle for the widespread implementation of prophylactic HPV vaccination of girls and adolescents via public health programs. HPV vaccination cannot reach soon enough the populations who need it the most, the very countries that bear a disproportionate burden of cervical cancer morbidity and mortality.

Financial \& competing interests disclosure

Eduardo Franco has served as occasional consultant to companies involved with human papillomavirus diagnostics (Gen-Probe, Roche and Qiagen), human papillomavirus vaccines (Merck and GSK), and cytology-based screening (Ikonisys and Cytyc). The author's entire research program has been funded by grants from Canadian Institutes of Health Research, US National Institutes of Health, Fonds de la Recherche en Santé du Québec, Cancer Research Society, and Canadian Cancer Society. The author has received salary awards from the Canadian Institutes of Health Research and Fonds de la Recherche en Santé du Québec. The author has no other relevant affiliations or financial involvement with any organization or entity with a financial interest in or financial conflict with the subject matter or materials discussed in the manuscript apart from those disclosed.

No writing assistance was utilized in the production of this manuscript. 